\title{
The Link between Barium Stars and Optically Bright Post-AGB Binaries
}

\author{
Hans Van Winckel ${ }^{1}$, Christoffel Waelkens ${ }^{1}$, Alain Jorissen ${ }^{2}$, Sophie Van Eck ${ }^{2}$, \\ Pierre North ${ }^{3}$ and Rens Waters ${ }^{4}$ \\ ${ }^{1}$ Instituut voor Sterrenkunde, K.U.Leuven, Belgium; ${ }^{2}$ Institut d'Astronomie, ULB, Belgium; \\ ${ }^{3}$ Institut d'Astronomie de l' Université de Lausanne, Switzerland; \\ " "Anton Pannekoek", VU Amsterdam and SRON Groningen, The Netherlands
}

Systematic radial velocity monitoring programs revealed that all optically bright, highlatitude post-AGB stars that show a near-IR excess in their energy distribution are binaries (see the contribution of R. Waters et al. in these proceedings). A standard AGB evolution for the shorter period objects seems, however, impossible since the orbits are too small to accommodate an AGB star! Non-standard phenomena connected to the specific binary nature have to be invoked in order to understand these systems.

In order to study the peculiar post-AGB binary stars in more detail, we started to investigate the relation with other classes of binary objects : the Ba giants (strong and mild), the Ba dwarfs and the Tc-poor S stars. These are chemically peculiar binary stars with one component being a cool old white dwarf. The chemical peculiarities were built up when the star, which is now the primary, accreted (by RLOF or wind-accretion) s-process elements enhanced material from the companion, which then was an AGB star (e.g.: McClure 1983, ApJ 268, 264; Jorissen and Mayor 1988, A\&A 198, 187).

1. The orbital periods found for the different groups are very similar. This suggests an evolutionary link between the post-AGB binaries and the Ba star family. Certainly the post-AGB binaries showing carbon-rich circumstellar material, are very good candidate progenitors of the $\mathrm{Ba}$ stars.

2. The eccentricities found for the short period post-AGB stars are higher than for the $\mathrm{Ba}$ giants, but similar to what is found for the $\mathrm{Ba}$ dwarfs. If the $\mathrm{Ba}$ dwarfs indeed evolve into Ba giants (North et al., 1996, IAU symp. 177, in press) this may suggest the eccentricity-distribution of the Ba giants is altered in a later evolutionary stage, for instance during the ascending of the red giant branch of the present giant. As the three groups of stars underwent AGB evolution of one of the components, the highly similar period and eccentricity distributions are determined probably by the same physical mechanisms. The formation of the shorter period systems remains, however, unexplained by current theory.

3. There are no extrinsic $S$ stars known so far with a period smaller than 600 days (Jorissen and Mayor 1992, A \& A, 260, 115). This cut-off period is much higher than the shorter periods found among the post-AGB stars.

A detailed analysis of the e- $\log (\mathrm{P})$ diagram of the different groups of evolved binary stars may enable us, not only to study the possible relation between the different binary groups, but also to study the binary interaction mechanisms and the evolution of the binary stars themselves. 